\title{
Formats des groupes et types de discussion dans la recherche sociale qualitative
}

\author{
Andrés Davila, Maître de conférences
}

Université du Pays Basque

Mario Domínguez, Maître de conférences

Université Complutense de Madrid

\begin{abstract}
Résumé
La dénomination «entretiens de groupe » renvoie à une pluralité de dispositifs de groupes de recherche. Compte tenu de la confusion qui est souvent malheureusement faite entre certains de ces dispositifs, cet article cherche à souligner l'intérêt que représente pour la recherche sociale qualitative le fait de mieux nuancer les différences entre l'entretien simultané, le focus group et le groupe de discussion, concrètement. C'est pourquoi cet article a pour objectif la prise en considération, tout à la fois, de sa configuration socio-historique (en ce qui concerne les entretiens simultanés pionniers ou focused interviews, par exemple), et de la forme distincte de la discussion que chacun met en scène (soit le débat soit la conversation), ainsi que de leurs dynamiques (semi-directive et progressive, pour le focus group, et non directive et processuelle, pour le groupe de discussion). Plaidant pour la possibilité d'une complémentarité des dispositifs plutôt que pour leur chevauchement.

Mots clés

RECHERCHE QUALITATIVE, PROCESSUS DE RECHERCHE, FOCUS GROUP, DÉBAT, GROUPE DE DISCUSSION, CONVERSATION, DISCOURS
\end{abstract}

\section{Introduction}

Un bref survol de la bibliographie spécialisée nous permet d'examiner l'usage qui y est fait de la dénomination "entretien de groupe » et en révèle le caractère polysémique. En fait, elle est utilisée indistinctement pour se référer à des méthodes de recherche sociale très dissemblables. Il est vrai que cette

RECHERCHES QUALITATIVES - Vol. 29(1), 2010, pp. 50-68.

ENTRETIENS DE GROUPE : CONCEPTS, USAGES ET ANCRAGES

ISSN 1715-8702 - http://www.recherche-qualitative.qc.ca/Revue.html

(C) 2010 Association pour la recherche qualitative 
dénomination fait référence au point commun à toutes ces méthodes consistant à travailler en situation d'entretien collectif, mais il est tout aussi vrai que l'utilisation généralisée de cette dénomination entraîne une confusion méthodologique croissante. Il n'est que de voir par exemple l'équivalence de plus en plus fréquente qui est faite entre focus group et groupe de discussion, comme si tout se résumait à la simple traduction idiomatique de deux dénominations pour un même instrument de collecte de données... au lieu d'aborder la manière selon laquelle l'un et l'autre sont constitués en tant que dispositifs de production de données.

C’est précisément sur ces aspects que nous voulons nous arrêter dans cet article, attentivement au fait que la distinction entre les spécificités de chacun de ces deux dispositifs de recherche est une condition nécessaire - quoique non suffisante - pour établir sa pertinence en vue de mettre en place une recherche. En outre, dans une telle recherche, les différents dispositifs pourraient aussi être concurremment utilisés, mais seulement lorsque nous saurons ce que chacun d'eux permet et empêche (ou, si l'on préfère, ce que chacun d'eux peut donner de lui-même). En fin de compte, ce n'est pas un type de dispositif précis qui confère sa forme à une recherche sociale qualitative, mais le processus même que celle-ci implique en fonction des divers moments qui la constituent. Et, dans cette optique, nous ne devrions pas perdre de vue le fait que, entre autres exigences, ce processus inclut l'adoption d'un positionnement « contre tout usage acritique des techniques de recherche" (Ortí, 1990, p. 154). En effet, aucun dispositif de recherche n'est innocent ou neutre, car chacun d'eux a sa propre constitution sociohistorique.

Pour pouvoir mettre en perspective la dénomination même d'entretien de groupe, il nous faut commencer par établir un point de référence commun à tous les lecteurs. Cependant, eu égard à l'hétérogénéité des situations et des dispositifs visés par telle ou telle mention de l'entretien de groupe, il n'est pas facile d'en choisir une qui soit capable de souligner les aspects qui le caractériseraient à chaque fois qu'il y est fait allusion, c'est-à-dire sans donner la prééminence à un dispositif plutôt qu'à un autre lorsqu'il s'agit de caractériser l'entretien de groupe.

C'est la raison pour laquelle nous reprenons ici cette caractérisation de Colette Baribeau, dans un article consacré à l'analyse de données obtenues par le biais de cette méthode : "L'entretien de groupe, comme son nom l'indique, suppose un groupe, un animateur et une discussion entre ces personnes " (Baribeau, 2009, p. 134). Le principal motif de notre choix réside dans le fait que cette caractérisation, aussi simple qu'évidente en apparence, rend compte des aspects communs aux divers dispositifs qui convergent sous cette 
dénomination, mais également de ceux qui les différencient: les types de discussion, de groupe, de dynamique... précisément. Au long des sections suivantes, nous examinerons chacune de ces différences à la lumière de certains dispositifs concernés par cette caractérisation.

\section{Au-delà de l'administration d'une série de questions... la discussion Discussion et recherche qualitative}

Il est indubitable que la discussion est présente dans les dispositifs groupaux de recherche, même si les types de discussion varient selon les cas. En fait, s'agissant de dispositifs de recherche sociale qualitative, les acceptions de l'action de « discuter » à prendre en considération sont trois en particulier :

- traiter attentivement et particulièrement une matière, oralement ou par écrit;

- considérer un sujet en le débattant;

- avoir une conversation sur un sujet.

Chacune indique une forme différente de la discussion qui se met en scène: l'examen, le débat et la conversation. À savoir trois situations d'énonciation précisément instituées par des dispositifs de recherche tels que l'entretien simultané, le focus group et le groupe de discussion, respectivement. Tout cela apparaît dès que l'on s'intéresse, même brièvement, à l'origine et à l'évolution de chacun d'eux.

Examinons la dénomination même de "groupe de discussion ", qui laisse percevoir les échos d'une autre époque, la seconde moitié des années quarante, où la désignation de «discussion groups" identifiait certaines expérimentations à caractère contrôlé, inspirées par les positions de Kurt Lewin; expérimentations établies pour l'étude de dynamiques de groupe, ainsi que pour la production de changement social en termes de formation et d'apprentissage dans les enseignements de base (Bradford \& French, 1948). Une dénomination à laquelle s'est toutefois rapidement substituée celle de T-group (abréviation de Training group), correspondant à ce que Lewin et son équipe ont mis en œuvre en 1947. Dénomination qui connaîtra alors un développement considérable dans le domaine de l'intervention psychosociologique au sein d'organisations et d'institutions tout d'abord aux États-Unis puis, à la fin la décennie suivante, en Europe. Au cours de l'époque qui a suivi la Seconde Guerre mondiale s'est également développée la psychothérapie de groupe, tant en raison de l'adoption psychanalytique de l'initiative, lancée en 1931-1932 par le médecin, philosophe et sociologue Jacob Levy Moreno (pionnier de l'approche psychodramatique ou de la sociométrie topologique), que de l'approche clinique (à la différence de 
l'approche psychosociale des T-groups) esquissée par Carl Rogers depuis les années cinquante sous la forme des « encounter groups » (Rogers, 1970).

Ces deux contextes de l'utilisation de dispositifs de groupes se révèlent déterminants au moment d'aborder la proposition et le développement du groupe de discussion pour la recherche sociale. Comme l'a si bien indiqué le sociologue Jesús Ibáñez dans sa vision des fondements, tant épistémologiques que méthodologiques, de ce type de groupe :

Le cœur de la dynamique de groupe se situe au niveau du groupe de travail (il tient compte du domaine social, mais pas du domaine libidinal), le cœur de la psychothérapie de groupe se situe au niveau du groupe de base (il tient compte du domaine libidinal, mais pas du domaine social) : le groupe de discussion est parfaitement équilibré, son cœur coïncide avec le centre de gravité au niveau de la bordure entre le groupe de base et le groupe de travail (il prend en compte l'articulation entre le domaine libidinal et le domaine social) (Ibáñez, 1981, p. 21).

Cette proposition d'un troisième contexte d'utilisation de ces dispositifs de groupes en recherche sociale plaide pour la construction d'un lieu pour une instance qui n'est plus extérieure ni intérieure, mais frontalière. Un lieu de rencontre, un espace et un temps conçus pour une tâche collective d'élaboration symbolique et inscrite dans le social : converser. Et la prise en charge, par ceux qui se constituent en membres de ce petit groupe, du travail commun consistant à entretenir une conversation, est précisément ce qui nous place dans un cadre d'interlocution non directive.

\section{De la focused interview au focus group}

Il est une autre situation énonciative, bien différente : celle qui suppose un débat. Cette situation d'énonciation propre au focus group présente des traits caractéristiques parmi lesquels nous retiendrons que, pour l'obtention de données, il est proposé de dépasser le domaine des interviews à questionnaires, encore que cette production de données reste envisagée du point de vue du jeu généralisé de questions-réponses qui y sont traitées. À cet égard, il ne faut pas oublier le fait qu'à l'origine du focus group se trouve précisément la focused interview, telle qu'envisagée par Robert Merton face au refus de plus en plus fréquent opposé par de "nombreux sujets, personnes et même des comportements et des questions sociales » au fait d'être "traités par le biais de moyennes statistiques » (Merton, Fiske \& Kendall, 1990, p. x). De fait, le début de ces groupes date de la fin de l'année 1941 lorsque, à la demande de Paul Lazarsfeld et de son équipe, Merton en programme l'utilisation comme un mécanisme complémentaire, pour mieux affiner, d'une part les réponses 
obtenues au préalable par des questionnaires sur des audiences radiophoniques -s'agissant de capter des motifs et des réflexions sur lesdites réponses de la part de ceux qui les avaient donnés -, et d'autre part l'évaluation de certains films produits pour remonter le moral et les convictions des soldats durant la $\mathrm{II}^{\mathrm{e}}$ Guerre mondiale.

Ses principes méthodologiques premiers figurent dans les interviews, sans codification des réponses, utilisées dans les épreuves de pré-test du questionnaire et de vérification de la qualité des données fournies dans les enquêtes. Des principes qui, tant en vue de peser sur des comportements collectifs déterminés que de favoriser un contexte social de persuasion de masse, allaient bientôt être appliqués à des groupes naturels, mais suivant la logique du "gain de temps et d'argent réalisé lorsque c'est un groupe de personnes qui est interviewé simultanément au lieu d'individus différents interviewés à différents moments » (Flick, 2004, p. 127). Une logique qui, au bas mot, rendrait sujette à caution la notion même de groupe par rapport à ce dispositif $^{1}$. C’est la raison pour laquelle nous avons opté ici pour la dénomination d' «entretien simultané », qui nous semble répondre plus précisément à ses caractéristiques.

Malgré cette continuité intellectuelle claire, les différences existant entre les deux types d'interviews, de groupe et individuels (Boutin, 1997, p. 34) n'ont pas tardé à se faire jour. En termes pragmatiques, l'interview de groupe s'est révélée beaucoup plus difficile à orienter vers des objectifs déjà prévus et, de même, les résultats obtenus échappaient fréquemment à leur traitement quantitatif ultérieur. Cependant, l'expansion de ces focused groups pionniers était plutôt due à l'utilisation qui en a été faite sous la pression des études de marché et la recherche de données comparables. Au point de considérer la redondance supposée des informations fournies par eux comme une donnée valable pour calculer la répartition des réponses; de sorte que, si plusieurs groupes répétaient une opinion, cela signifierait que ladite opinion est amplement partagée. Par ailleurs, outre le fait de multiplier le nombre de sujets interrogés, les focused groups (dénommés par la suite focus groups) présentaient divers avantages par rapport aux entretiens individualisés, ainsi celui de permettre de multiples définitions d'une même situation, ou des réponses plus diversifiées, voire innovantes, par rapport au guide préétabli.

En réalité, l'intervention de chaque participant établit implicitement un modèle pour le reste, de façon à fournir des réponses de plus en plus personnalisées, ce qui a pour résultat de structurer le débat sous forme d'un flux circulant dans une direction grâce au relâchement successif des inhibitions (Merton, Fiske \& Kendall, 1990, p. 143). Il faut réaffirmer que, contrairement à 
ce qui arrive dans une situation d'entretien simultané ou focused interview (dont les participants ne génèrent qu'une série d'interventions individuelles dans un contexte collectif d'écoute), dans une situation de focus group il y a reconnaissance mutuelle des autres en termes de co-construction énonciative; cependant, sa structure telle qu'elle se présente pèse encore trop sur l'appréhension des aspects propres de l'interaction entre intervieweur et interviewé (effet halo, influence, etc.), incarnés ici dans les figures du modérateur et des participants du groupe.

À titre de résumé, nous présentons dans le Tableau 1 quelques-unes des caractéristiques distinctives mentionnées jusqu'à présent à propos de chacun des dispositifs, classés par degré d’ouverture du plus petit au plus grand.

\section{Le guide, le scénario et le groupe (soit " centré ", soit de discussion)}

Face à l'amalgame peu regardant qui est souvent fait, dans nombre d'articles et de manuels de recherche sociale qualitative, entre les dispositifs de focus group et de groupe de discussion, il nous semble particulièrement bien approprié de proposer une comparaison synthétique entre ces deux dispositifs en tenant compte de certains de leurs traits les plus caractéristiques. Nous nous proposons ici de contester l'estompage des différences entre focus groups et groupes de discussion. De plus en plus fréquente dans la pratique de recherche, la confusion des deux formulations empêche d'envisager l'éventualité même (ainsi que les différents modes) de leur complémentarité. Ce n'est pas sans raison que dans l'utilisation de focus groups priorité est généralement donnée à une orientation plutôt instrumentale, axée vers l'obtention de données tandis que, dans le cas de groupes de discussion, l'orientation est plutôt critique, axée sur la compréhension des processus sociaux en cours. Même si les deux orientations doivent être présentes, à un degré plus ou moins fort et selon le cas, dans la réalisation de chaque recherche concrète.

\section{Du guide de débat pour un focus group...}

À cet effet, il n'est pas inutile de commencer par rappeler ce qui est exprimé dans la dénomination même de focus group : «focaliseur » désigne un appareil ou dispositif servant à focaliser; c'est-à-dire, concentrer, rassembler. Ainsi, un faisceau lumineux se focalise en concentrant un rayonnement en un point, le « foyer ». Est «focal» ce qui concerne un foyer: le foyer d'un instrument d'optique, les foyers d'un cône... le foyer de feu; car enfin, focal et foyer dérivent du latin classique focus : «feu », donc le lieu central et symbolique du foyer dans les pièces d'une maison, et que l'on retrouve encore dans la traduction de focus group par "groupe centré ». Une exigence permanente de focalisation qui, en tout cas, retombe sur la personne qui contrôle le groupe. Rien d'étonnant donc à l'effort consacré à ce sujet dans la bibliographie spécia- 
Tableau 1

Formats de groupes et types de discussion

\begin{tabular}{llll}
\hline $\begin{array}{l}\text { Formats de } \\
\text { groupes }\end{array}$ & $\begin{array}{l}\text { Entretien } \\
\text { simultané }\end{array}$ & Focus group & $\begin{array}{l}\text { Groupe de } \\
\text { discussion }\end{array}$ \\
\hline $\begin{array}{l}\text { Types de } \\
\text { discussion }\end{array}$ & $\begin{array}{l}\text { Confrontation } \\
\text { d'opinions par le } \\
\text { biais de } \\
\text { l'administration } \\
\text { individualisée d'une } \\
\text { série de questions } \\
\text { qui doivent recevoir } \\
\text { des réponses en } \\
\text { public. }\end{array}$ & $\begin{array}{l}\text { Un ensemble de } \\
\text { discussions (sous } \\
\text { forme d'échanges } \\
\text { successifs } \\
\text { d'arguments } \\
\text { divers) focalisées }\end{array}$ & $\begin{array}{l}\text { Une seule } \\
\text { discussion } \\
\text { (provoquée en } \\
\text { vane d'une } \\
\text { interaction semi- } \\
\text { recherche } \\
\text { concrète) mais } \\
\text { ouverte au cours } \\
\text { de son } \\
\text { développement } \\
\text { non directif } \\
\text { Conversation }\end{array}$ \\
& Examen & Débat & \\
\hline $\begin{array}{l}\text { Situation } \\
\text { d'énonciation }\end{array}$ & & &
\end{tabular}

lisée (comme Krueger, 1994 ou Greenbaum, 1998, par exemple), qui se distingue par son ardeur à déterminer les caractéristiques propres à un bon modérateur ou animateur, mais aussi par la préoccupation envers tout ce qui touche à la préparation et au déroulement d'une série de discussions dans une même session.

C’est dans cette perspective que s'est élaboré le guide pour un débat. En définitive, le débat est le mode adopté par toute discussion faite de récapitulatifs partiels, en vue de faire un bilan final. C'est pour cela que, dans un tel guide, il faut examiner l'introduction des différents thèmes à traiter, avec leurs respectives questions préétablies (tant en termes d'influence que d'attribution), ainsi que le matériel qui pourrait être requis à cet égard dans chaque cas. Il s'agit donc d'une construction linéaire et continue, par épisodes, de tel dispositif groupal de recherche. Construction chronologique qui apparaît manifeste chaque fois que l'on pose comme critère de référence que «le modèle recommandé pour débuter la discussion de groupe comporte quatre étapes : 1 . La bienvenue; 2 . Le rappel du thème à traiter; 3 . Les règles de base; 4. La première question » (Krueger, 1994, p. 113). Il en résulte que l'évaluation de la consistance interne et du degré d’imprécision des réponses figure parmi les préoccupations de base à propos de l'information obtenue par ce biais.

La Figure 1 montre la mise en forme du focus group comme une succession de plans étagés en profondeur (a, b, c...) avec l'objectif de parvenir 


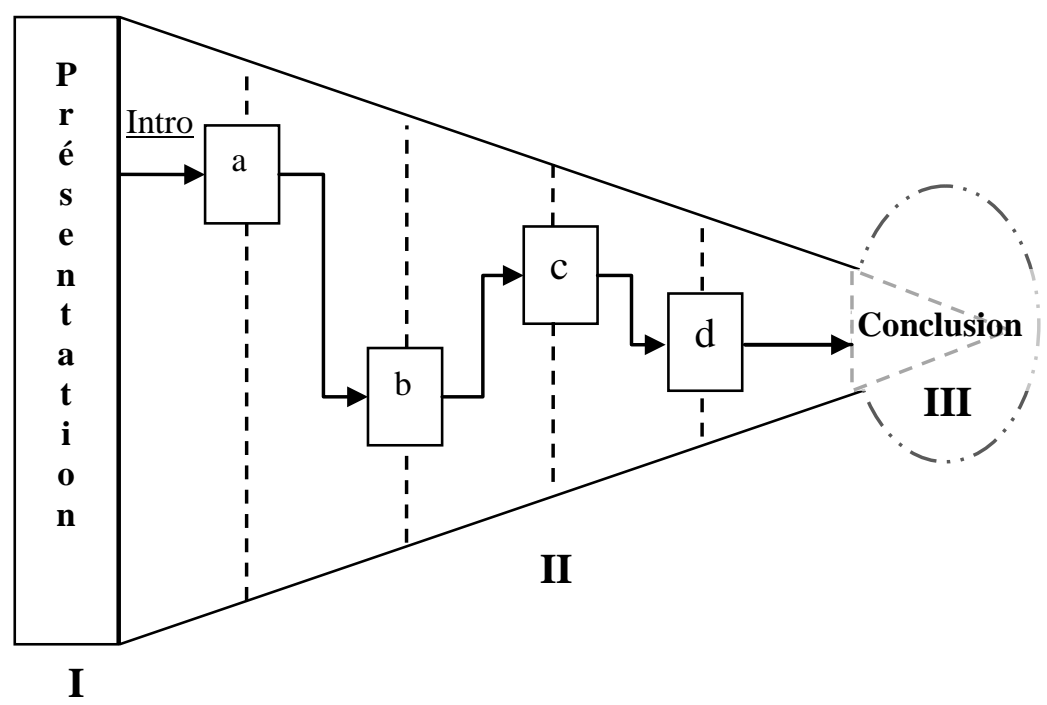

Figure 1 : Stratégie du GUIDE de débat (topic guide) pour un focus group

à une conclusion concertée par l'intermédiaire d'une dynamique semidirective. Pour les modérateurs qui animent les discussions de groupe, il faut mettre en balance les opinions des (3 à 6) participants du débat, répertorier les thèmes qui ont été discutés, et chercher à obtenir le consensus.

\section{...au scénario de conversation pour un groupe de discussion}

Dans le groupe de discussion priment cependant la constitution et la dissolution du groupe moyennant la conversation entre ses (6 à 9) participants. Si ceux-ci peuvent s'entretenir de manière informelle, c'est que, au contraire du focus group, il ne s'agit pas de diriger la dynamique du groupe en respectant une grille d'entrevue ni de maintenir les débats à l'intérieur de frontières établies d'avance. Il s'agit de provoquer une conversation orientée. Le groupe de discussion se caractérise ainsi par le développement non-directif d'une discussion génératrice de signification, et non seulement d'information. D'où l'importance qu'acquiert l'élaboration de la stratégie à suivre dans chaque cas par l'intermédiaire d'un scénario schématique de conversation, qui peut être divisé en trois sections fondamentales : 
I. l'examen des modes selon lesquels seront présentées tout à la fois la recherche et ceux qui la conduisent, ainsi que la session et ceux qui y participent, toutes ces présentations devant être en consonance avec les aspects préalablement traités pendant la prise de contact;

II. la détermination de la manière dont on souhaite susciter la conversation de la part du groupe; à savoir, une provocation initiale qui devra être cohérente avec ce qui a déjà été exposé tant au cours de la prise de contact que lors des présentations, et qui devra également être capable de provoquer une dynamique ouverte de conversation (généralement dénommée « discours libre »);

III. l'élaboration d'un ordre logique (et non chronologique ou selon des séquences à suivre par le groupe) des diverses situations, des diverses thématiques, des diverses questions... impliquées par les objectifs concrets de la recherche, en vue de les aborder dans la conversation de groupe et lors des provocations (tant initiale que de relance). En effet, le scénario rend compte de la stratégie d'urgence et de l'induction à la discussion de celui qui assume la figure de précepteur du groupe (la personne qui prend en charge la dynamique du groupe sans y prendre part).

La Figure 2 schématise la vocation à intégrer le devenir conversationnel (c'està-dire les manières de discourir et de dériver du groupe), caractéristique du scénario pour un groupe de discussion, par opposition à l'évocation d'un ordre de débat préétabli (que le groupe doit respecter progressivement), propre au guide du focus group.

\section{Les moments dans le processus de recherche qualitative}

À travers cette confrontation entre l'une et l'autre technique de groupe, esquissées ici avec les Figures 1 et 2, nous voudrions pointer une distinction trop souvent négligée entre une orientation plus progressive du focus group et l'orientation nettement processuelle du groupe de discussion.

Il est indubitable que la confusion habituelle entre processus et progrès, au point de les considérer comme synonymes, n’aide pas vraiment à souligner cette distinction. Dans chaque processus nous pourrons apprécier des moments, chacun d'eux comportant non seulement ce qui est susceptible de contribuer à sa mise en marche (progrès), mais aussi à sa mise en question (Davila, 2007). Il s'agit là d'une différenciation importante si l'on considère qu'une recherche sociale qualitative est constituée sous la forme d'un processus de recherche ouvert. Un type de processus qui est assumé de manière radicale par la technique du groupe de discussion. 


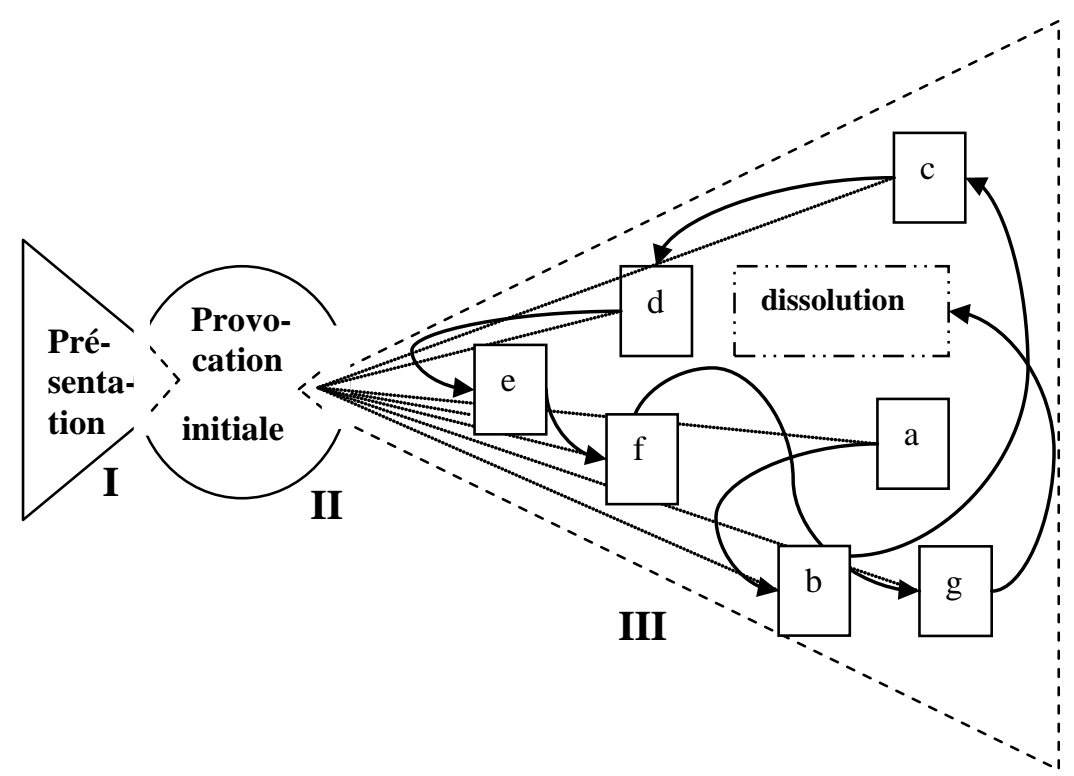

Figure 2 : Stratégie du SCÉNARIO de conversation pour un Groupe de discussion

La Figure 3 montre jusqu'à quel point le processus est ouvert, versus la version par étapes qui est typique de la recherche quantitative, tant en ce qui concerne le design (qui «sera modifié en fonction des événements imprévus qui surviennent tout au long du processus ») que l'analyse ("dans la mesure où peuvent se produire des informations non prévues dans le design »); tant en ce qui concerne les dispositifs (d'où la dénomination plus appropriée d' « entrevue ouverte ", justifiée par son caractère non directif, au lieu d' « entrevue en profondeur ») que les personnes qui les constituent - en intégrant le sujet de la recherche, conçu comme un sujet en processus -, face à l'insistante disjonction entre sujet et objet opérée par le paradigme classique (Ibáñez, 1986b, p. 72-78). Paradigme à partir duquel une recherche sociale se constitue uniquement comme appareil de capture dont la méthode est la reproduction itérative, et dont les opérations fondamentales (réaction, disjonction et réjection, décrites par Edgar Morin) se réunissent sous la dénomination de "paradigme de simplification » (Morin, 1981). À cet égard, il nous faut souligner le fait que l'invention du groupe de discussion répond à un paradigme complexe pour la recherche sociale (Davila, 1997), dans lequel il s'inscrit en tant que pratique conversationnelle avant que d'être une réunion de groupe. 

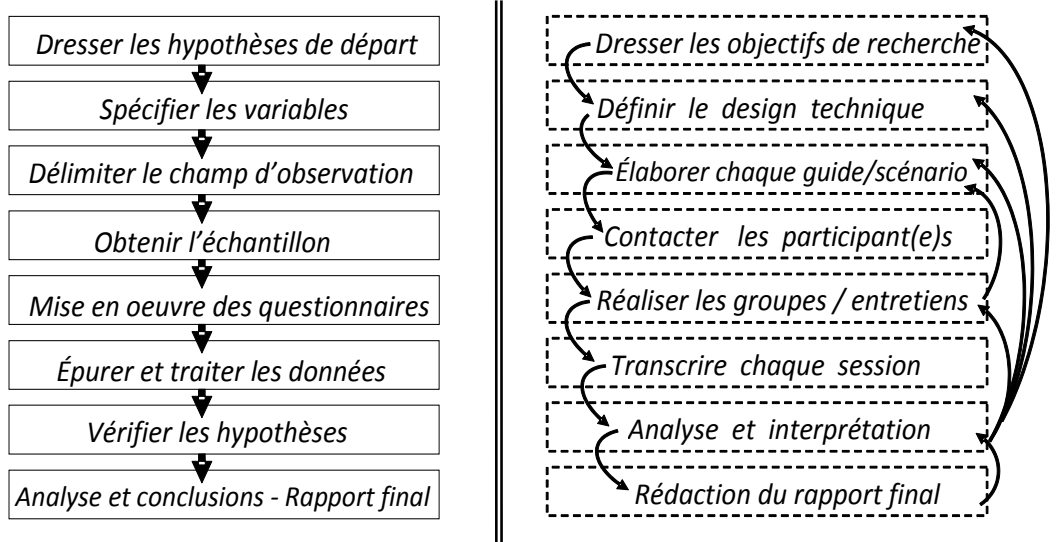

Figure 3 : Conformation d'une recherche sociale en étapes ou en boucles (Davila , 2007, p. 187)

\section{Spécificités du groupe de discussion : pratique de la conversation et discours}

\section{La pratique de la conversation dans le processus de recherche}

La conversation, «cette relation sociale élémentaire » et « tout à fait négligée par les sociologues ${ }^{2} »$ (Tarde, 1989, p. 7), se donne tant comme la forme par antonomase de la rencontre (converser, c'est, avant tout, se fréquenter, ce qui était le sens propre du latin conversari) que comme dialogue dans toute son ambivalence (en le modelant en termes à la fois de réciprocité et de divergence). Aussi avons-nous tendance à oublier qu'une dénomination comme celle de " réunion de groupe » se réfère au groupe de base et qu'en aucun cas elle ne pourrait rendre compte d'un dispositif tel que celui du groupe de discussion, où « la provocation est délimitée par la formation du groupe et la proposition d'un thème de discussion, alors que l'écoute n'est délimitée que par ce qui est pertinent pour ce thème » (Ibáñez, 1986b, p. 76).

Ce qui est propre à un groupe de discussion, c'est la génération d'une conversation - et d'une seule - entre les membres du groupe, et non pas la prolifération de plusieurs conversations, distinctes et croisées, qui rendrait impossible le processus de production du groupe lui-même. D'où l'importance, tant du design de la recherche et de l'échantillonnage, que de la transcription lorsque nous travaillons en groupes de discussion. Le design et l'échantillonnage permettent de rendre compte de ceux qui doivent entretenir la 
conversation (structure et formation de chaque groupe de discussion), tandis que sa transcription fait apparaître ses moments constitutifs. Développons maintenant brièvement ces deux aspects :

Commençons par le design de la recherche et la caractérisation de ceux qui doivent entretenir la conversation, en rappelant que, dans la formulation du groupe de discussion, « la conversation est un tout : un tout qui est plus que la somme de ses parties, qui ne peut pas se répartir en interlocuteurs ni en (inter)locutions - c'est pourquoi c'est l'unité minimale. Chaque interlocuteur est non pas une entité, mais un processus : en conversant, il change, tout comme change également le système dans lequel il converse » (Ibáñez, 1991, p. 77).

Et c'est précisément pour que personne ne cesse de parler, ou ne parle en fonction de ce qu'il croit être la pensée des autres (restreignant ainsi la conversation sous l'effet de préjugés et d'idées préconçues sur les autres), que le groupe de discussion se compose habituellement de personnes qui n'avaient pas de relations proches ou fréquentes, afin de minimiser les sous-entendus habituels entre personnes qui se connaissent déjà. De fait, le dispositif du groupe de discussion nous éloigne du groupe naturel, au point de se révéler dès sa propre constitution comme un groupe simulé et manipulable :

- simulé, car il s'agit d' « un groupe purement imaginaire, un groupe qui ne devient groupe qu'en espérance... car [ses participants/membres] ne pourront être réunis que pendant qu'ils parlent [et] l'espace de la réunion ne leur sera assigné que pour le temps de la discussion » (Ibáñez, 1986a, p. 271);

- et manipulable, car le chercheur (ou précepteur) «a en main tous les fils qui meuvent le groupe. Il a le pouvoir de leur assigner l'espace et, en contrôlant la durée de cette assignation, de contrôler leur temps. Mais il a également le pouvoir de déterminer le groupe : il fixe le nombre et les personnes qui vont venir » (Ibáñez, p. 272), en fonction des objectifs de recherche fixés.

Loin de l'assimiler à un tout homogène et stable en le naturalisant, il s'agit, en traçant ses frontières (taille, durée, dynamique, composition, etc.), de permettre l'objectivation symbolique des processus sociaux par lesquels « tout groupe vit des compromis qu'il invente et des contradictions qu'il génère (jusqu'à des seuils au-delà desquels il ne peut pas les assumer) " (Certeau, 1994, p. 249). C'est la raison pour laquelle, au moment d'établir les caractéristiques sociales qui constituent la composition d'un groupe de discussion, il nous faudra tenir compte de l'homogénéité et de l'hétérogénéité de ce groupe, donc de ses profils définis à partir des caractéristiques sociales 
prises en considération dans le design technique de la recherche, dans chaque

cas particulier. À partir de la variable sexe, par exemple, on peut délimiter un groupe homogène au cas où tous les membres du groupe seraient hommes ou femmes; d'un autre côté, on peut délimiter un groupe hétérogène si le groupe est composé d'hommes et de femmes. Le profil d'un groupe peut être homogène selon telle variable et, en même temps, hétérogène selon telle autre. Ainsi un groupe composé d'hommes et de femmes, tous enseignants, est-il un groupe homogène à l'égard de la variable « emploi » et hétérogène à l'égard de la variable « sexe ».

La conception et la sélection de l'échantillon composé par les profils de groupes choisis permettent de construire l'espace social de référence de la recherche concrète dans lequel s'inscrivent les groupes de discussion.

À cet égard, le choix exclusif de groupes de discussion, tout comme l'utilisation complémentaire de focus groups avec des groupes de discussion, nous permettront, en vertu de similitudes et de différences constitutives, d'analyser les différentes positions socio-discursives qui configurent l'espace social de référence à étudier.

Dans une recherche sociale qualitative, pour dessiner l'échantillon de la recherche, on procède par saturation des positions de l'espace social de référence. Les critères de choix ne sont donc pas l'étendue - puisque la représentativité statistique n'intervient pas ici - mais la pertinence et l'adéquation aux objectifs de recherche (Plummer, 1983). Ainsi, s'agissant d'un échantillon structurel et non statistique, il convient de déterminer le minimum technique pertinent des groupes requis pour chaque étude correcte afin de pouvoir obtenir, par l'analyse, des régularités significatives au lieu d'une redondance répétitive. Prenons, par exemple, le cas d'une recherche sur les représentations sociales de la forêt. Le fait d'établir l'échantillon à partir de divers profils de groupes du secteur forestier (travailleurs, propriétaires, etc.) nous permet de confirmer la répétition de stéréotypes (tel que le cas de la portée de l'image de la forêt en tant que poumon); mais aussi bien nous permet de détecter l'émergence d'autres images qui, bien qu'elles surgissent rarement, nous apportent des clés importantes pour l'analyse (tel que le cas de la fonction dépurative de la forêt à partir de l'image du rein). Le simple fait de constater ces déplacements de sens (le rein à l'égard du poumon) nous place dans en autre registre d'interprétation des activités qui donnent forme à ce monde social (Shibutani, 1955).

\section{Le groupe de discussion, la conversation et le discours}

Au contraire du focus group dont la dynamique coupe le discours de groupe, le groupe de discussion est une technique de recherche qui favorise dudit discours 
à travers de la conversation réalisée. De cette manière, à notre avis, le groupe de discussion est une technique spécialement adéquate pour étudier l'objet de recherche à partir d'une perspective qu'envisage la délimitation, l'analyse et l'interprétation des divers positions socio-discursives présentes dans ledit objet.

Il convient de clarifier la distinction entre le discours produit par chaque sujet énonciateur du groupe (discours comme produit de l'énonciation de chaque sujet membre du groupe) et le «discours de groupe » (discours comme produit de la conversation du groupe); ce qui devient indubitablement confus en raison de l'utilisation fréquente d'expressions telles que le «discours du groupe » qui laisse entendre que le groupe produit " un discours » alors que ce qui est produit à travers le groupe est "du discours ", c'est-à-dire des potentialités discursives qui feront l'objet d'une distinction dans l'analyse, le moment venu. C'est un processus qui suppose l'échange de pratiques signifiantes, ce qui, dans le cas du groupe de discussion, implique d'interpréter et d'analyser les conventions significatives, linguistiques et non linguistiques, qui y ont cours.

Pour cela, il est crucial de passer par la transcription de toutes et chacune des sessions. Transcription qui, il faut le souligner, doit être littérale de sorte que, dans chacune d'elles, nous mentionnerons tous les marqueurs linguistiques (jusqu'aux signes de ponctuation) et paralinguistiques (donc, identifiant chaque personne intervenante, et enregistrant les intonations, les rires, les gestes, les silences, les pauses, le temps, etc.) nécessaires pour rendre compte de ce déplacement de l'oral à l'écrit. Le fait de consigner tous ces éléments dans le texte nous permettra de le comprendre dans son sens le plus complet, en dotant de sens le texte alors considéré comme produit d'une situation d'énonciation spécifique. En effet, la clé du processus d'analyse est une lecture et relecture du texte, moment par moment, bloc par bloc, mot par mot...; (re)lecture associée à un sens critique de la part de la personne qui mène la recherche, ce qui impliquera des questionnements répétés concernant la manière dont on parle d'une affaire : Est-ce la seule manière possible? Y a-t-il d'autres façons de dire la même chose? Pourquoi est-ce cette forme qui est utilisée ici et maintenant et pas une autre? etc. Il s'agit donc de travailler à la fois le niveau explicite de ce qui se dit dans le groupe et le niveau implicite ou, si l'on préfère, le non-dit que nous analyserons à partir de constructions particulières de la phrase, de certaines intonations (qui dénotent ironie ou sarcasme, par exemple), de telle ou telle réticence dans la parole... et que seule la transcription littérale nous restitue. N'oublions pas que la conversation caractéristique du groupe de discussion est la «conversation en zigzag " (Moeschler, 1984, p. 168), et que chacun des réaménagements demande que soient indiquées les conditions de production des énoncés, ce qui nous révèle jusqu’à quel point ce qui se dit sera 
aussi important que le fait de savoir quand, pourquoi, dans quel but, pour qui, comment et jusqu'à quel point cela se dit.

Au contraire de la conception linéaire du discours dans lequel chaque locuteur (ou énonciateur unique chaque fois) contrôle, du début à la fin, ce qui est énoncé, la transcription nous révèle que le discours subit des déviations continuelles (digressions, parenthèses...) ainsi que des changements de direction qui inscrivent ce qui est énoncé dans une interaction où l'énoncé est interrompu, démenti, modifié, etc. La transcription exprime l'orientation du discours, à la fois en lui attribuant une fin déterminée et en rendant visible son propre déploiement temporel, en un jeu permanent d'anticipations («je te l'expliquerai plus tard...») et de retours en arrière («mais je t’ai déjà dit avant... »); allées et venues propres à la forme d'action qu'implique le discours même, terme qui dérive de discurrere ou action de parcourir en tous sens ${ }^{3}$.

Le profil de chaque groupe doit être élaboré en tenant compte du fait que, pour qu'une conversation se fasse dans un groupe de discussion, il faudra que les relations entre leurs participants soient symétriques, de sorte que les relations de domination qui peuvent exister dans l'espace social de référence ne se reproduisent pas dans le groupe, car, dans le cas contraire, il ne serait tout simplement pas possible de constituer un tel groupe et il n'y aurait donc ni conversation, ni discours. Au lieu de quoi, le groupe de discussion est constitué par un groupe de sujets qui sont énonciateurs de discours dans le déroulement de la conversation (la notion de discours s'entendant alors comme le produit d'une situation énonciative); et le groupe de discussion a pour caractéristique propre le fait que, en tant que dispositif de recherche, les discours produits dans la conversation concrète de chaque groupe font partie, à leur tour, de la production de discours.

N'oublions pas que parler est déjà en soi une manière d'agir sur l'autre, en impliquant celui qu'écoute dans ce qui se dit, au point d'en faire un coénonciateur et non plus un simple destinataire, d'où le fait que, dans tout récit, le développement (discursif) même s'accuse autant, voire plus important que son contenu (informatif). Sans aller plus loin, l'émergence d'une métaphore dans un groupe peut donner le départ à toute une ligne conversationnelle, avec un résultat des plus fructueux pour l'analyse.

En effet, ce n'est pas seulement la métaphore de départ qui présente une importance, mais également le fait que ce soit précisément la dynamique conversationnelle qui renforce la possibilité, pour le cadre métaphorique, de se poursuivre, de se développer et de nous fournir des clés interprétatives importantes pour l'analyse. Prenons, à titre d'exemple, la situation dans laquelle l'un des participants à un groupe de discussion introduit, à propos de 
l'obligation d'apprendre et connaître une langue minoritaire, la métaphore de l'acquisition d'un billet de train pour pouvoir monter dans le train; le reste des participants du groupe reprend l'image et se développent des questions telles que l'obligation, l'imposition, le besoin... de l'apprentissage de cette langue, en partant de l'utilisation d'éléments comme le voyage en train, l'achat d'un billet, la fonction du contrôleur du train, les endroits par lesquels le train passe, etc. Au bout de compte, avoir une conversation avec quelqu'un implique de (se) convaincre ${ }^{4}$.

\section{Notes}

${ }^{1}$ A l'instar de ce qui se produit avec d'autres dispositifs dont la dénomination inclut un appel au groupe, comme dans le cas du "groupe nominal » où, de l'avis de Lorraine Savoie-Zajc, "l'appellation "groupe" est trompeuse car il s'agit en fait d'un rassemblement d'individus qui n'ont que très peu d'interaction verbale entre eux. Leurs messages sont médiatisés par l'animateur du groupe, d'où l'appellation de "nominal" » (Mucchielli, 1996, p. 88).

${ }^{2}$ Même plus d'un siècle après que Gabriel Tarde a émis cet avis, avec des exceptions comme celle d'Everett C. Hughes et Mark Benney, pour lesquels «chaque conversation possède son propre équilibre entre révélation et dissimulation des pensées et des intentions : c'est seulement dans des circonstances très inhabituelles que les paroles sont si complètement explicites que chaque mot doit être pris à sa valeur faciale [le modèle de l'échange d'informations entre scientifiques] » (Hughes \& Benney, 1996, p. 282).

3 Pour une approche intéressante du discours, pour la recherche sociale, voir Charaudeau et Maingueneau, 2002; Maingueneau, 1991.

${ }^{4}$ Comme nous l'indiquait déjà Montaigne : « L'art de converser n’est pas un art de convaincre mais bien au contraire : un art d'être convaincu. L'étymologie même du terme l'explicite : L'art de conférer est littéralement l'art de céder, de passer la parole à l'autre et de lui donner raison, de se vaincre soi-même en se pliant devant l'évidence d'autrui » (Navarro, 2007, p. 291).

\section{Références}

Baribeau, C. (2009). Analyse des données des entretiens de groupe. Recherches Qualitatives, 28(1), 133-148.

Boutin, G. (1997). L'entretien de recherche qualitatif. Ste-Foy: Presses de l’Université de Québec.

Bradford, L.P., \& French, J.R.P. (Éds) (1948). The dynamics of the discussion group. Journal of Social Issues 4, 1-75. 
Certeau, M. de (1994). L'actif et le passif des appartenances. Dans M. Certeau (Éd.), La prise de parole et autres écrits politiques (pp. 248-268). Paris : Seuil.

Charaudeau, P., \& Maingueneau, D. (2002). Dictionnaire d'analyse du discours. Paris : Seuil.

Davila, A. (1997). Apuntes acerca de una construcción (que en Jesús Ibáñez es y se hace) compleja para la investigación social. Dans F. Álvarez-Uría (Éd.), Jesús Ibáñez. Teoría y práctica (pp. 135-152). Madrid : Endymion.

Davila, A. (2007). Representaciones sociales e investigación social cualitativa. Dans F.J. Cerrato, \& A. Palmonari (Éds), Representaciones sociales y psicología social : comportamiento, globalización y posmodernidad (pp. 185-204). Valencia : Editorial Promolibro.

Flick, U. (2004). Introducción a la investigación cualitativa. A CoruñaMadrid : Fundación Paideia Galiza-Ediciones Morata.

Greenbaum, Th.L. (1998). The handbook for focus group research. Thousand Oaks : Sage.

Hughes, E.C., \& Benney, M. (1996). La sociologie et l'entretien. Dans E.C. Hughes, Le regard sociologie. Essais choisis (pp. 281-290). Paris : École des Hautes Études en Sciences Sociales.

Ibáñez, J. (1981). Usos tópicos y abusos utópicos de las técnicas de grupo. Revista de la Asociación Española de Neuropsiquiatría, 2, 16-36.

Ibáñez, J. (1986a). Más allá de la sociología. El grupo de discusión : Técnica y crítica ( $2{ }^{\mathrm{e}}$ éd.). Madrid : Siglo XXI.

Ibáñez, J. (1986b). Perspectivas de la investigación social : El diseño en las tres perspectivas. Dans M. Ferrando, J. Ibáñez, \& F. Alvira (Éds) El análisis de la realidad social. Métodos y técnicas de investigación. Madrid: Alianza Universidad Textos.

Ibáñez, J. (1991). El regreso del sujeto. La investigación social de segundo orden. Santiago. Chili : Editorial Amerindia.

Krueger, R.A. (1994). Focus groups. A practical guide for applied research ( $2^{\mathrm{e}}$ éd.). Thousand Oaks : Sage.

Maingueneau, D. (1991). L'analyse du discours. Introduction aux lectures de l'archive. Paris : Hachette.

Merton, R.K., Fiske, M., \& Kendall, P.L. (1990). The focused interview : a manual of problems and procedures ( $2^{\mathrm{e}}$ éd.). New York : Free Press. 
Moeschler, J. (1984). Argumentation et conversation. Éléments pour une analyse pragmatique du discours. Paris : Hatier-Credif.

Morin, E. (1981). La Nature de la nature. Paris : Le Seuil.

Mucchielli, A. (1996). Dictionnaire des méthodes qualitatives en sciences humaines et sociales. Paris : Armand Colin.

Navarro Reyes, J. (2007). Pensar sin certezas. Montaigne y el arte de conversar. México : Fondo de Cultura Económica.

Ortí, A. (1990). La apertura y el enfoque cualitativo o estructural : la entrevista abierta y la discusión de grupo. Dans M. Ferrando, J. Ibáñez, \& F. Alvira (Éds), El análisis de la realidad social. Métodos y técnicas de investigación. Madrid : Alianza Universidad Textos.

Plummer, K. (1983). Documents of life. An introduction to the problems and literature of a humanistic method. London : Georges Allen \& Unwin.

Rogers, C.R. (1970). Carl Rogers on encounter groups. New York : Harper \& Row.

Shibutani, Th. (1955). Reference groups as perspectives. American Journal of Sociology, 60, 522-529.

Tarde, G. (1989). L'Opinion et la foule. Paris : PUF. Document consulté le 7 mars 2009 de

http://classiques.uqac.ca/classiques/tarde_gabriel/opinion_et_la_foule/tarde _opinion_et_la_foule.pdf

Andrés Davila est maître de conférences au Département de Sociologie 2, Faculté des Sciences Sociales et de la Communication, Université du Pays Basque (UPV/EHU). Responsable de l'Atelier de Recherche Sociale Qualitative (KOA) et chercheurmembre du Groupe des Études pour la Cohésion Sociale (KOHESLAN). En particulier, il s'intéresse aux méthodologies qualitatives et à la modélisation dans la recherche qualitative et à l'analyse du discours, avec contributions à diverses thématiques de recherche: les conditions de production et de réception des représentations sociales au sujet des minorités défavorisées et migrantes, de l'activité agricole...; la configuration des imaginaires urbains par l'intermédiaire des médias (photographie, notamment); ou l'institutionnalisation et la socialisation des formes sécuritaires, par exemple. Ses projets de recherche en cours portent notamment sur l'innovation sociale dans un contexte de développement durable (en prenant en considération pour cela l'inclusion sociale, l'environnement et la santé).

Mario Domínguez est docteur en sociologie par l'Université Complutense de MadridUCM (1998) et maître de conférences à la même Université. Il est également diplômé 
68 RECHERCHES QuALITATIVES / Vol. 29(1), 2010

en géographie et histoire (1986) et en sociologie (1990). Ses domaines de recherche incluent la théorie sociologique, la sociologie des sciences, la sociologie politique, les mouvements politiques et les conflits sociaux. Il a participé, entre autres, dans les suivantes projets de recherche et développement ( $R \& D)$ : la mondialisation et les mouvements sociaux, les processus de socialisation dans des conditions extrêmes, l'analyse des théories sociales sur l'aide sociale, et des études sur le développement durable. Il a également participé dans plusieurs publications scientifiques et techniques et à des conférences sur des sujets tels que l'émergence des jeunes contrevenants, les modèles de la réadaptation sociale ou la perception de soi des jeunes squatters en Espagne. 